\title{
VALIDATION OF TEST METHODS FOR AIR LEAK RATE VERIFICATION OF SPACEFLIGHT HARDWARE
}

\author{
Heather A. Oravec \\ The University of Akron \\ Akron, Ohio, USA
}

\author{
Christopher C. Daniels \\ The University of Akron \\ Akron, Ohio, USA
}

\author{
Janice L. Mather \\ The University of Akron \\ Akron, Ohio, USA
}

\section{ABSTRACT}

As deep space exploration continues to be the goal of NASA's human spaceflight program, verification of the performance of spaceflight hardware becomes increasingly critical. Suitable test methods for verifying the leak rate of sealing systems are identified in program qualification testing requirements. One acceptable method for verifying the air leak rate of gas pressure seals is the tracer gas leak detector method. In this method, a tracer gas (commonly helium) leaks past the test seal and is transported to the leak detector where the leak rate is quantified. To predict the air leak rate, a conversion factor of helium-to-air is applied depending on the magnitude of the helium flow rate. The conversion factor is based on either the molecular mass ratio or the ratio of the dynamic viscosities. The current work was aimed at validating this approach for permeation-level leak rates using a series of tests with a silicone elastomer O-ring. An established pressure decay method with constant differential pressure was used to evaluate both the air and helium leak rates of the O-ring under similar temperature and pressure conditions. The results from the pressure decay tests showed, for the elastomer O-ring, that neither the molecular flow nor the viscous flow helium-to-air conversion factors were applicable. Leak rate tests were also performed using nitrogen and argon as the test gas. Molecular mass and viscosity based helium-to-test gas conversion factors were applied, but did not correctly predict the measured leak rates of either gas. To further this study, the effect of pressure boundary conditions was investigated. Often, pressure decay leak rate tests are performed at a differential pressure of $101.3 \mathrm{kPa}$ with atmospheric pressure on the downstream side of the test seal. In space applications, the differential pressure is similar, but with vacuum as the downstream pressure. The same O-ring was tested at four unique differential pressures ranging from 34.5 to $137.9 \mathrm{kPa}$. Up to six combinations of upstream and downstream pressures for each differential pressure were compared. For a given differential pressure, the various combinations of upstream and downstream dry air pressures did not significantly affect the leak rate. As expected, the leak rate of the O-ring increased with increasing differential pressure. The results suggested that the current leak test pressure conditions, used to verify spacecraft sealing systems with elastomer seals, produce accurate values even though the boundary conditions do not model the space application.

Keywords: conversion factor, helium leak detector, leak rate, permeation, pressure decay, verification

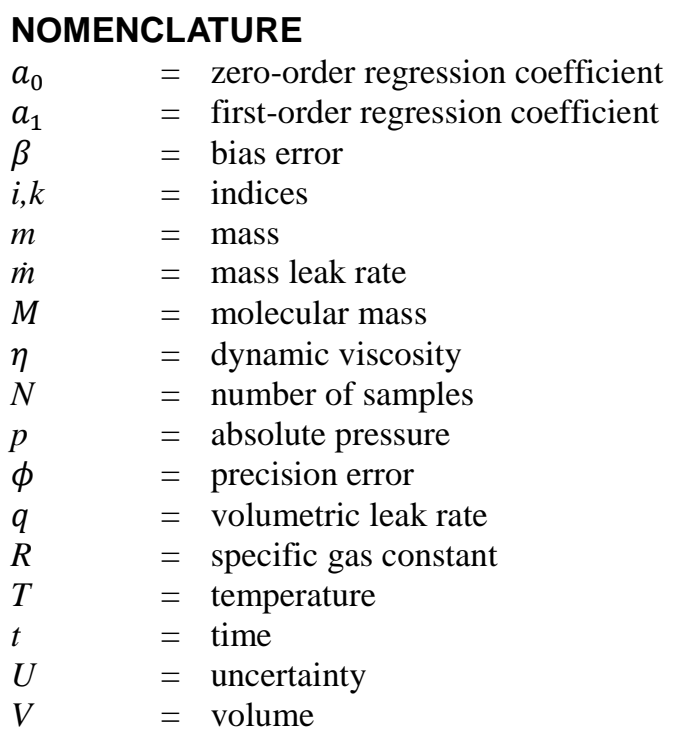

\section{INTRODUCTION}

The success of any spaceflight mission is dependent on the verification program set forth to ensure that systems and components meet the specifications and requirements defined at the outset of the program. As NASA has its sights on deep space exploration, especially manned missions, verification of hardware performance is imperative. Acceptable verification methods include analyses, demonstration, inspection, test, or any combination thereof [1]. To verify the leak rates of pressurized units or sealing systems, testing is the verification method most 
commonly utilized. Acceptable leak test methods are outlined in program requirements [2,3]. For meaningful results, the selected test method must accurately simulate the functional conditions of the unit under test following the test as you fly, fly as you test approach [1]. For sealing systems, this may include the operational temperature range, pressure conditions, gas type, and exposure to unique space environment elements (e.g., atomic oxygen, ultraviolet radiation, and foreign object debris). In some instances, the test method is selected based on project resources, schedule, and feasibility and therefore, may not incorporate all aspects of the operating conditions. In other instances, the test method itself may not be capable of simulating the operating conditions. In either case, flawed data resulting from poorly simulated or misapplied test methods could be misinterpreted as a valid representation of the hardware performance. In this paper, the soundness of leak test methods used to verify the air leak rate of spacecraft sealing systems is investigated.

Two common methods to evaluate the leak rate of gas pressure seals are the tracer gas leak detector method and the pressure change or pressure decay technique. These methods have long been used to quantify the leak rates of silicone elastomer seals used in habitat type space applications, e.g., docking system seals [4-10]. The tracer gas leak detector method is often a preferred test method due to its sensitivity to small leaks and relatively short test time. With this method, a chemically inert tracer gas, typically helium, is used to pressurize the volume upstream of the seal under test. The downstream side is placed under vacuum and connected to a mass spectrometer leak detector. As the helium migrates downstream of the test article, it enters the mass spectrometer, which ionizes the gas particles, and then separates the ions based on their molecular weight. A volumetric flow rate is then associated with the collection of helium ions.

For small leaks down to $10^{-12}$ sccs, the helium leak detector method is highly accurate and the test itself can be completed in short order, although time and manpower are expended in the calibration procedure and required post-processing of the test data. Another drawback of this method is that for sealing systems that must perform to an air leak rate requirement (e.g., docking hatch seals) the helium leak rate must be converted to a mass flow rate of air - a process that is not as obvious as it may seem. The conversion from volumetric to mass flow rate is trivial, and is achieved by multiplying the volumetric flow rate by the density of the test gas; however, the conversion from helium to air is more involved and often misapplied.

Data sheets with conversion factors that can be applied to the measured helium leak rate often accompany the documentation included with a helium leak detector [11]. In practice, helium is converted to air by applying a factor of 0.374 to the measured volumetric leak rate following Eqn. 1:

$$
q_{\text {air }}=\sqrt{\frac{M_{H e}}{M_{\text {air }}}} * q_{\mathrm{He}},
$$

where, the square root of the molecular mass ratio of helium-toair is equal to the conversion factor ( 0.374 in this case). This value is derived from the volumetric flow rate of a gas through an orifice in the molecular flow regime [12,13]. Similar conversion factors have been established based on viscous flow using the ratio of dynamic viscosities (e.g., $\eta_{H e} / \eta_{\text {air }}$ ). Standard conversion factors for the viscous and molecular flow regimes are listed in Table 1 for some common gases.

For a leak detected in a space sealing system, the determination of the flow regime can be challenging. Generally, the flow regime is estimated by the ratio of the average mean free path to the diameter of the leak path-otherwise defined as the Knudsen number. This estimation assumes that a pore leak with physical dimensions, such as a crack, hole, or other material defect is present within the sealing system [14]. It does not account for a leak dominated by gas permeation through the seal material, as is the case with silicone elastomer space seals $[15,16]$. Currently, there is no universal conversion factor, based upon theory, for the helium-to-air leak rate conversion of permeation dominant systems. This is due, in part, to the variations in compounding different elastomers and the installed configuration of the elastomer in different systems. To date, no work has compared the permeation dominated leak rates of air and helium to determine a ratio.

Table 1. Standard helium leak rate conversion factors for viscous and molecular flow regimes [11].

\begin{tabular}{ccc}
\hline \hline & \multicolumn{2}{c}{ Multiply Helium Leak Rate by: } \\
\cline { 2 - 3 } Convert to: & Viscous Flow & Molecular Flow \\
\hline Nitrogen & 1.12 & 0.374 \\
Air & 1.08 & 0.374 \\
Argon & 0.883 & 0.316 \\
\hline
\end{tabular}

Besides the tracer gas leak detector method, another commonly accepted leak test method is the pressure decay technique with mass point leak rate analysis. The pressure decay technique does not require any conversions since air is utilized as the test gas. In this method, a known volume of pressurized gas permeates through and/or leaks at the interface of the seal under test while the pressure and temperature are recorded with time [17]. The Ideal Gas Law is applied to calculate the mass of the gas $\left(m_{i}\right)$ in the known volume at each time-step $\left(t_{i}\right)$. A linear least-squares regression, centered about the differential pressure of interest, is used to find the best-fit line to the mass-time dataset. The mass flow rate of the test article is defined by the first-order coefficient $\left(a_{1}\right)$ of the best-fit line.

Though the pressure decay method is low-cost and applicable to an extensive range of leak rates, it has several limitations that reduce feasibility. First, the size of the internal volume and the magnitude of the leak, among other factors, determine the test duration. The combination of large volume and small leak may result in a test that spans several days costing time and manpower. Conversely, large leaks from a small volume may occur in such a short time that the limited amount of collected data leads to large measurement uncertainty. Second, although the method accounts for the temperature of the gas in the calculation of the leak rate, the location of the measurement is critical and correct sensor placement can be difficult in certain 
testing environments.

Additionally, leak tests are typically performed at a differential pressure of $101.3 \mathrm{kPa}$. To achieve this, the lowpressure region is either placed under vacuum or the ambient air pressure is utilized. Ambient pressure is more commonly used to avoid the inclusion of a secondary seal in the hardware design. However, when ambient pressure is used, changes in barometric pressure may extend the test duration and/or invalidate the leak rate measurement. More critically, the ambient downstream pressure does not simulate the vacuum of space. It is well known that differential pressure is a driving factor for the final leak rate value, but there are no reports on the effect of varying the upstream and downstream pressure boundary conditions which generate the differential pressure.

As such, the purpose of this study was twofold, but focused on the verification of a spacecraft sealing system's air leak rate using the helium leak detector method and the pressure decay method. The first objective was to prove or disprove that the conversion of the measured helium leak rate to an equivalent air (or other gas) leak rate could be accomplished using standard conversion factors. The second objective was to investigate the effect of pressure boundary conditions on the air leak rate when using the pressure decay method of testing. Using a modified pressure decay method, a series of leak tests was performed on a silicone elastomer O-ring. All tests in this series were completed at or near room temperature and with a differential pressure of $101.3 \mathrm{kPa}$, applying vacuum downstream of the test article. Four different gases including helium, nitrogen, dry air, and argon were utilized in this series. From the experimental data, conversion factors were computed for helium to each test gas and compared to the industry standards for viscous and molecular flow. A second series of leak tests was conducted at four unique differential pressures ranging from 34.5 to $137.9 \mathrm{kPa}$. Dry air was the test gas for all tests in this series. For each differential pressure, up to six combinations of unique upstream and downstream pressures were tested and the final leak rate values were compared. The experimental set up, methodology, and test results for each test series are discussed herein.

\section{DESCRIPTION OF EXPERIMENTS}

In this study, two series of leak rate experiments were performed using a modified pressure decay method. Four test sets were completed in the first series: one set for each test gas. Four repeats per test set were performed for a total of sixteen tests. Eighteen tests were completed in the second series with no repeats. Most tests were conducted at ambient room temperature $\left(20^{\circ} \mathrm{C}\right)$; however, some were run within an environmental chamber controlled to $23^{\circ} \mathrm{C}$. The slight difference in temperature was accounted for in the data analysis and did not affect the final results. Additional specifics of the experimental setup and test methodology are detailed in the following sections.

\section{Test Article}

A single test article was evaluated throughout this study. The test article was made from high temperature silicone multipurpose O-ring cord stock. The cord stock had a nominal cross-sectional diameter of $9.5 \mathrm{~mm}$ and durometer Shore A hardness of 70. The cord stock was cut to length and the two ends bonded with Loctite ${ }^{\circledR}$ Superflex ${ }^{\circledR}$ Clear RTV Silicone Sealant (\#59530) to form one continuous test article. The test article had a nominal $30.5-\mathrm{cm}$ outer diameter and was sized to fit in the groove of a custom aluminum test fixture. Once installed, the test article remained in the test fixture, physically undisturbed, for the duration of the study.

\section{Test Gases}

In the first test series, a total of four different inert gases were used to evaluate the leak rate of the test article. These included helium, nitrogen, dry air, and argon. Dry air was used as the test medium to evaluate the leak rate under various pressure boundary conditions in the second test series. The specifications for all test gases are listed in Table 2.

Table 2. Test gas specifications.

\begin{tabular}{ccccc}
\hline \hline Component & Helium & Nitrogen & Dry Air & Argon \\
\hline $\mathrm{He}$ & $99.999 \%$ & -- & -- & -- \\
$\mathrm{N}_{2}$ & -- & $99.999 \%$ & -- & -- \\
$\mathrm{Ar}$ & -- & -- & -- & $99.998 \%$ \\
\hline $\mathrm{O}_{2}$ & $<2 \mathrm{ppm}$ & $<2 \mathrm{ppm}$ & $19.5-$ & $<5 \mathrm{ppm}$ \\
$\mathrm{Moisture}$ & $<2 \mathrm{ppm}$ & $<3 \mathrm{ppm}$ & $<3 \mathrm{ppm}$ & $<5 \mathrm{ppm}$ \\
$\mathrm{THC}$ & $<0.5 \mathrm{ppm}$ & $<0.5 \mathrm{ppm}$ & $<0.5 \mathrm{ppm}$ & $<2 \mathrm{ppm}$ \\
\hline $\mathrm{CO}_{2}$ & -- & -- & $<1 \mathrm{ppm}$ & - \\
$\mathrm{CO}$ & -- & -- & $<1 \mathrm{ppm}$ & -- \\
\hline
\end{tabular}

\section{Pressure Boundary Conditions}

In the first test series, all tests were run with a differential pressure of $101.3 \mathrm{kPa}$. In general, the upstream pressure was 1.3 times atmospheric pressure. Vacuum was applied downstream of the test article.

To determine the effect of pressure boundary conditions on the leak rate of the test article in the second series, four individual test sets were run - each set with a unique differential pressure. Within each test set, the differential pressure was held constant, but the initial upstream pressure was varied per test. The different pressure combinations for each test set are shown in Table 3. These pressure conditions were selected to be within the limitations of the measurement transducers used in the test assembly.

Table 3. Pressure boundary conditions for dry air leak rate tests on the silicone elastomer $\mathrm{O}$-ring test article.

\begin{tabular}{ccccc}
\hline \hline & Set 1 & Set 2 & Set 3 & Set 4 \\
\hline Differential pressure, $\mathrm{kPa}$ & 34.5 & 68.9 & 103.4 & 137.9 \\
\hline & 68.9 & -- & -- & -- \\
Initial upstream pressure, & 103.4 & 103.4 & -- & -- \\
$\mathrm{kPa}$ & 137.9 & 137.9 & 137.9 & - \\
& 172.4 & 172.4 & 172.4 & 172.4 \\
& 206.8 & 206.8 & 206.8 & 206.8 \\
& 241.3 & 241.3 & 241.3 & 241.3 \\
\hline
\end{tabular}




\section{Test Method}

A modified pressure decay method was used to measure the leak rate of the test article in this study. This method was similar to the standard pressure decay method with mass point leak rate analysis, but used a control system to maintain the desired differential pressure across the test article. Previous work has shown that this enhanced method is accurate, reliable, can be used to measure both large and small leaks, minimizes test time, and improves the measurement uncertainty [18].

In this method, the test apparatus, Fig. 1, consisted of a hermetically sealed volume of gas on the upstream side of the test article. The pressurized volume of gas was allowed to leak downstream of the test article into a region of lower pressure. This low-pressure region was controlled to maintain a constant differential pressure across the test article throughout the test duration. A differential pressure transducer was used to measure the pressure difference between the high- and low-pressure regions. A controller monitored the differential pressure and compared it to the chosen set point value. As the differential pressure varied from the set point, due to permeation or interface leakage, the controller reacted by sending a voltage signal to a pressure regulator. The pressure regulator appropriately raised or lowered the downstream pressure through connections of vacuum and ambient pressure. In cases where the downstream pressure was above ambient pressure (refer to Table 3), the regulator was connected to a gas supply system.

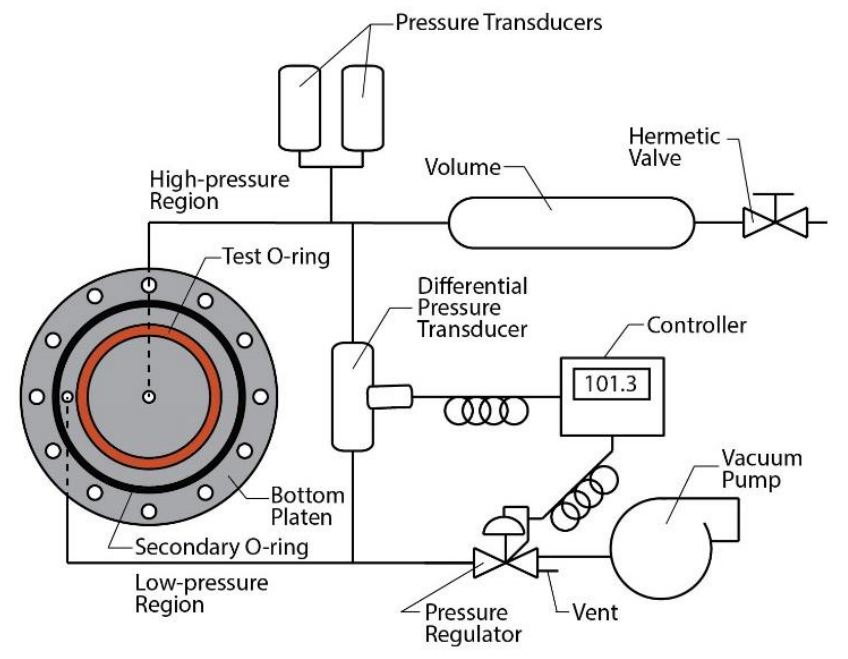

Figure 1. Schematic of leak rate test apparatus with controlled downstream pressure (in cases where downstream pressure was above ambient, the regulator was connected to a gas supply system instead of vacuum).

Not unlike the standard pressure decay method, the pressure and temperature of the gas in the sealed volume were recorded with time. Following the assumptions of the Ideal Gas Law, the mass of gas within the volume was calculated at each time-step $\left(t_{i}, m_{i}\right)$ using Eqn. 2.

$$
m=p V / R T
$$

In this equation, the volume, $V$, was determined in advance through application of Boyle's Law.

Assuming a constant leak rate, a linear least-squares regression was computed to determine the best-fit line to the dataset. Unlike the standard pressure decay method, all data could be included in this computation since a constant differential pressure was maintained. The best-fit line was modeled by Eqn. 3, where the first-order coefficient, $a_{1}$, represented the mass leak rate $(\dot{m})$ of the test article.

$$
m(t)=a_{1} t+a_{0}
$$

The measurement uncertainty of the leak rate was calculated using the generalized Eqn. 4 [17,19].

$$
\begin{aligned}
U_{\dot{m}}^{2}= & \sum_{i=1}^{N}\left(\frac{\partial \dot{m}}{\partial m_{i}}\right)^{2} \beta_{m_{i}}^{2}+\sum_{i=1}^{N}\left(\frac{\partial \dot{m}}{\partial t_{i}}\right)^{2} \beta_{t_{i}}^{2} \\
& +\sum_{i=1}^{N}\left(\frac{\partial \dot{m}}{\partial m_{i}}\right)^{2} \phi_{m_{i}}^{2}+\sum_{i=1}^{N}\left(\frac{\partial \dot{m}}{\partial t_{i}}\right)^{2} \phi_{t_{i}}^{2} \\
& +2 \sum_{i=1}^{N-1} \sum_{k=i+1}^{N}\left(\frac{\partial \dot{m}}{\partial m_{i}}\right)\left(\frac{\partial \dot{m}}{\partial m_{k}}\right) \phi_{m_{i} m_{k}} \beta_{m_{i}} \beta_{m_{k}} \\
& +2 \sum_{i=1}^{N-1} \sum_{k=i+1}^{N}\left(\frac{\partial \dot{m}}{\partial t_{i}}\right)\left(\frac{\partial \dot{m}}{\partial t_{k}}\right) \phi_{t_{i} t_{k}} \beta_{t_{i}} \beta_{t_{k}} \\
& +2 \sum_{i=1}^{N-1} \sum_{k=i+1}^{N}\left(\frac{\partial \dot{m}}{\partial t_{i}}\right)\left(\frac{\partial \dot{m}}{\partial m_{k}}\right) \phi_{t_{i} m_{k}} \beta_{t_{i}} \beta_{m_{k}}
\end{aligned}
$$

Assuming no errors in the measurement of time, and using correlation coefficients that produce maximum uncertainty [19], the previous equation can be reduced to Eqn. 5

$$
U_{\dot{m}}^{2}=\sum_{i=1}^{N}\left(\frac{\partial \dot{m}}{\partial m_{i}}\right)^{2} \beta_{m_{i}}^{2}+\sum_{i=1}^{N}\left(\frac{\partial \dot{m}}{\partial m_{i}}\right)^{2} \phi_{m_{i}}^{2}
$$

The partial derivative of $\dot{m}$ with respect to $m_{i}$ is:

$$
\frac{\partial \dot{m}}{\partial m_{i}}=\frac{N t_{i}-\sum_{i=1}^{N} t_{i}}{N \sum_{i=1}^{N}\left(t_{i}^{2}\right)-\left(\sum_{i=1}^{N}\left(t_{i}\right)\right)^{2}}
$$

And the bias and precision errors, respectively, are:

$$
\begin{gathered}
\beta_{m}^{2}=\left(\frac{V}{R T} \beta_{p}\right)^{2}+\left(\frac{p}{R T} \beta_{V}\right)^{2}+\left(\frac{p V}{R^{2} T} \beta_{R}\right)^{2}+\left(\frac{p V}{R T^{2}} \beta_{T}\right)^{2} \\
\phi_{m}^{2}=\left(\frac{V}{R T} \phi_{p}\right)^{2}+\left(\frac{p}{R T} \phi_{V}\right)^{2}+\left(\frac{p V}{R^{2} T} \phi_{R}\right)^{2}+\left(\frac{p V}{R T^{2}} \phi_{T}\right)^{2}
\end{gathered}
$$

As shown, the bias and precision errors include contributions from the measurement instruments which were obtained though the instruments' calibration records, product specifications, or computations. 
Using the modified pressure decay method, the test article's leak rate and measurement uncertainty were calculated in realtime.

\section{Test Apparatus}

The complete test apparatus consisted of the test fixture with hermetic plumbing, gas supply system, measurement instrumentation, differential pressure control system, and data acquisition (DAQ) hardware and associated software. The test fixture consisted of two clear anodized platens manufactured from 6061-T6 aluminum. The test article was installed into a recessed grove in the bottom platen, constrained only along the outer diameter of the O-ring, Fig. 2. The O-ring was free to move inward, however, once the upper platen was installed and the interior volume was pressurized, movement in this direction was not anticipated. The upper platen was installed onto the lower platen compressing the O-ring by $17 \%$ of its nominal crosssectional diameter.

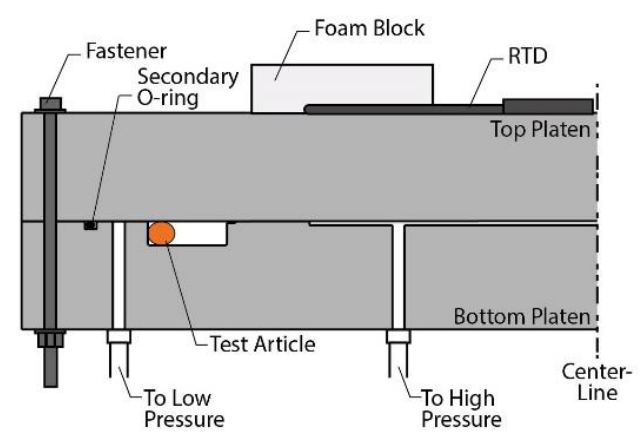

Figure 2. Schematic of leak rate test fixture cross-section.

The test gas was supplied to the high-pressure side of the test apparatus to the desired initial pressure ranging from 68.9 to $241.3 \mathrm{kPa}$, refer to Pressure Boundary Conditions. The lowpressure side was controlled to achieve the desired differential pressure set-point value which ranged from 34.5 to $137.9 \mathrm{kPa}$. A secondary O-ring of larger inside diameter was installed in the test fixture, concentric to the test article, such that the pressure downstream of the test article could be increased or reduced as necessary.

To determine the mass of gas at each time-step (Eqn. 2), the measured pressure, volume, and temperature were required. The gas pressure in the high-pressure region was measured using two pressure transducers whose values were averaged by the data acquisition system. This average value was used in the data processing. For reference, typical bias and precision errors of the pressure transducers were 15.6 $\mathrm{Pa}$ and $12.0 \mathrm{~Pa}$, respectively.

The volume of the high-pressure region changed over the course of the study due to slight modifications in the fixture plumbing. For each modification, the volume was directly measured using a minimum of 31 applications of Boyle's Law where $p_{1} V_{1}=p_{2} V_{2}$. The total volume changed from $234.3 \pm 3.8 \mathrm{~mL}$ to $286.2 \pm 6.6 \mathrm{~mL}$ depending upon the configuration. For the corresponding tests, the appropriate volume was used in the computations for leak rate and did not affect the overall results of the study.

The temperature of the gas in the high-pressure region was indirectly measured using a resistance temperature detector (RTD). The RTD was placed on the upper platen and insulated with a foam block to minimize changes in the temperature readings due to laboratory conditions. The RTD had Class A accuracy and typical bias and precision errors of $0.196^{\circ} \mathrm{C}$ and $0.0225^{\circ} \mathrm{C}$, respectively. Recall that some tests were run in an environmental chamber controlled to $23^{\circ} \mathrm{C}$. Other tests were conducted in the ambient laboratory environment. For these tests, the temperature reading did not vary by more than $\pm 2.1^{\circ} \mathrm{C}$ per test, which negligibly impacted the results. The representative temperature for the ambient laboratory tests was $20^{\circ} \mathrm{C}$.

The data acquisition system consisted of signal conditioners and an associated computer software program. The DAQ was used to collect the pressure and temperature measurements at a nominal rate of $10 \mathrm{~Hz}$. These values were combined with the volume measurement in the computer software program to calculate the mass of the test gas at each time-step. The software also calculated the test article's leak rate and associated measurement uncertainty in real-time. In general, each test ran continuously for a maximum duration of 29 hours unless otherwise manually stopped.

\section{TEST RESULTS AND DISCUSSION}

The results of this study are discussed in the following sections. The first section summarizes the results of the first test series investigating the applicability of using standard heliumto-air conversion factors for verifying air leak rates. The second section presents the findings of the second test series investigating the effect of pressure boundary conditions used in the pressure decay method.

\section{Validation of Conversion Factors}

In the first test series, the internal volume of gas was pressurized to approximately 1.3 times atmospheric pressure, and the downstream pressure was controlled to maintain a constant differential pressure of $101.3 \mathrm{kPa}$. The leak rates of helium, nitrogen, dry air, and argon through the silicone elastomer test article were compared. For each gas, the leak test was repeated four times. The test results were highly repeatable providing confidence in the test method, Fig. 3. The argon results displayed the greatest scatter with a maximum difference of $6.7 \times 10^{-12} \mathrm{~kg} / \mathrm{s}$ between repeat tests. The average mass leak rate values for each gas are plotted in Fig. 4. The error bars represent the measurement uncertainty. As shown, the leak rate increased with the molecular mass of the test gas.

The leak rate of the O-ring was also measured using a helium leak detector. The average volumetric leak rate, calculated from four repeat tests, was $1.03 \times 10^{-4} \operatorname{sccs}( \pm 5.7 \%)$. This value was converted to a mass flow rate of $1.84 \times 10^{-11} \mathrm{~kg} / \mathrm{s}$. The average helium leak rate measured using the modified pressure decay method, Fig. 4 , was $1.81 \times 10^{-11} \mathrm{~kg} / \mathrm{s}$, a difference of $1.6 \%$. This comparison provided an additional level of 
confidence in the measured results using the modified pressure decay test method.

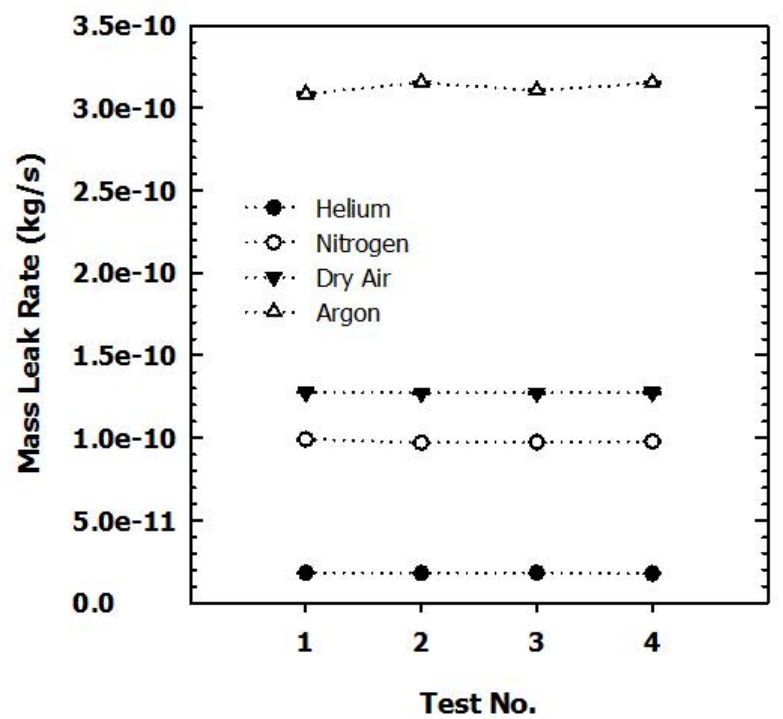

Figure 3. Repeat leak test results for a silicone elastomer O-ring tested with four different gases using a modified pressure decay method. Error bars represent measurement uncertainty.

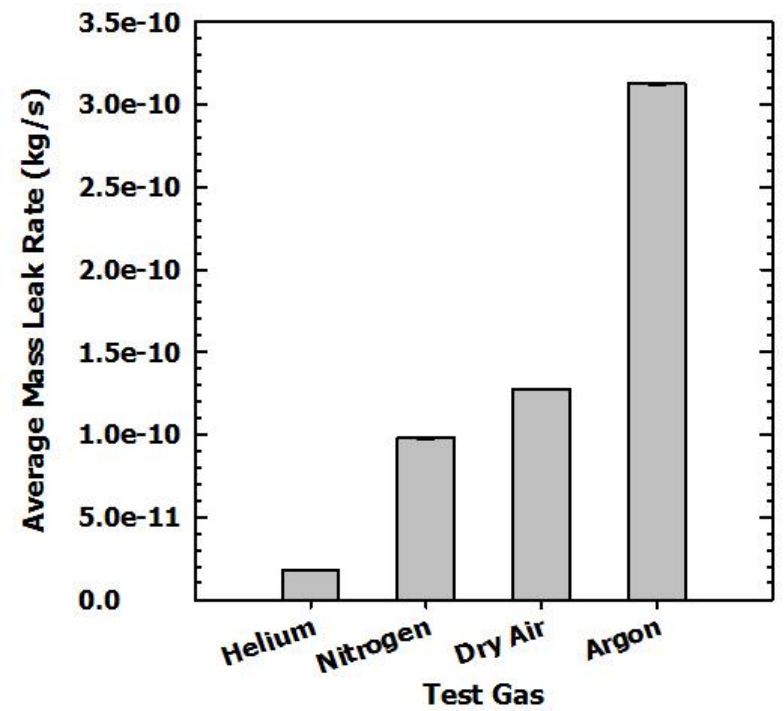

Figure 4. Average experimental leak rate of a silicone elastomer O-ring for four different gases, measured using a modified pressure decay method. Error bars represent measurement uncertainty.

The average mass flow rates of helium, nitrogen, dry air, and argon from the modified pressure decay tests were converted to volumetric flow rates at normal temperature and pressure (NTP: $\left.20^{\circ} \mathrm{C}, 101.3 \mathrm{kPa}\right)$. For example, the average mass flow rate of helium $\left(1.81 \times 10^{-11} \mathrm{~kg} / \mathrm{s}\right)$ was converted to a volumetric flow rate of $1.09 \times 10^{-4} \mathrm{~cm}^{3} / \mathrm{s}$ (NTP). Experimental conversion factors (test gas-to-helium volumetric flow ratios) were computed and are shown in Table 4 with the standard viscous and molecular flow regime conversion factors for comparison. As can be seen, the experimental conversion factors did not align with the standard values for either flow regime.

Table 4. Experimental helium-to-test gas conversion factors compared to standard conversion factors for viscous and molecular flow regimes.

\begin{tabular}{cccc}
\hline \hline \multirow{2}{*}{ Convert to: } & \multicolumn{3}{c}{ Multiply Helium Leak Rate by: } \\
\cline { 2 - 4 } & $\begin{array}{c}\text { Experimental } \\
\text { Ratio }\end{array}$ & Viscous & Molecular \\
& 0.77 & 1.12 & Flow \\
\hline Nitrogen & 0.97 & 1.08 & 0.374 \\
Air & 1.73 & 0.883 & 0.374 \\
Argon & & 0.316 \\
\hline
\end{tabular}

Furthermore, the standard viscous and molecular flow factors for each gas were applied to the measured volumetric flow rate of helium to calculate the projected nitrogen, air, and argon leak rates. Figure 5 displays the experimental leak rate values measured for each gas compared to the projected values. No comparison was needed for helium-to-helium; therefore, only one bar is shown. As expected, neither the application of the viscous flow factor nor the molecular flow factor to the measured helium leak rate correctly predicted the nitrogen, dry air, nor argon leak rates of the test article. When molecular flow was assumed, the converted leak rates consistently underpredicted the leak rate of the test article. When viscous flow was assumed, the converted leak rates for nitrogen and dry air were overpredicted, but the leak rate for argon was underpredicted.

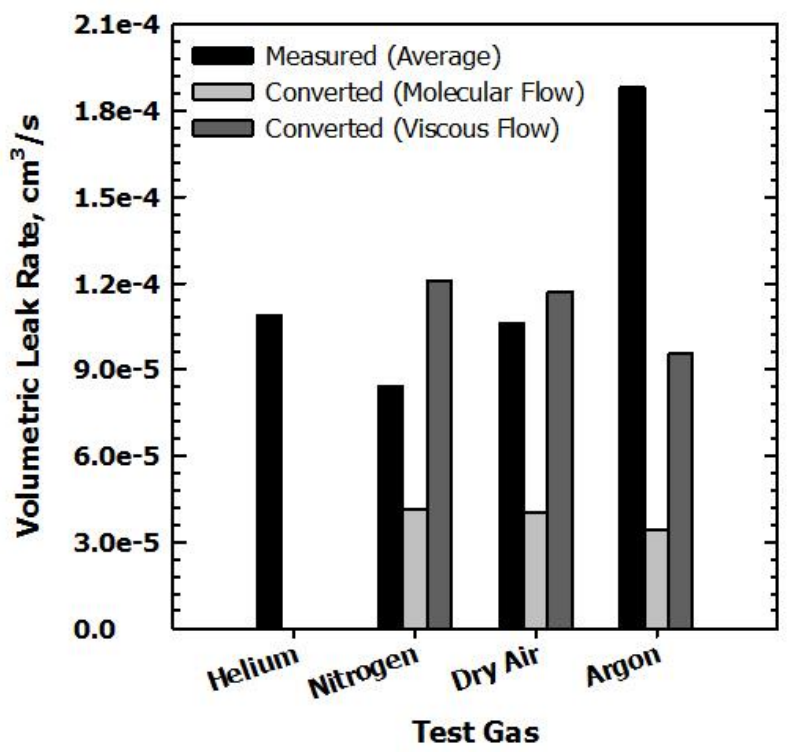

Figure 5. Experimental average volumetric leak rates at NTP compared to projected leak rates of nitrogen, dry air, and argon computed by applying standard conversion factors for molecular and viscous flow to the measured helium leak rate. 
In a similar fashion, the average volumetric leak rates for nitrogen and argon were converted to air leak rates in the viscous and molecular flow regimes. The standard nitrogen-to-air and argon-to-air conversion factors applicable for the molecular flow regime were calculated using the radical in Eqn. 1 with the molecular mass of nitrogen and argon substituted for that of helium (e.g., $\sqrt{M_{A r} / M_{\text {air }}}$ ). The standard conversion factors for the viscous flow regime were calculated using the ratios of the dynamic viscosities of nitrogen-to-air and argon-to-air (e.g., $\left.\eta_{\text {Ar }} / \eta_{\text {air }}\right)$. Figure 6 shows the experimentally measured dry air leak rate compared to the projected air leak rate values computed by applying the standard conversion factors to the measured helium, nitrogen, and argon leak rate values. No conversion was needed for air-to-air; therefore, only one bar is shown. The conversion from helium-to-air in the viscous flow regime most closely represented the measured air leak rate, but overpredicted the value by $11 \%$. In the molecular flow regime the leak rate was underpredicted by $62 \%$. The conversion from nitrogen-to-air underpredicted the measured leak rate in both flow regimes. Conversely, the conversion from argon-to-air overpredicted the leak rate.

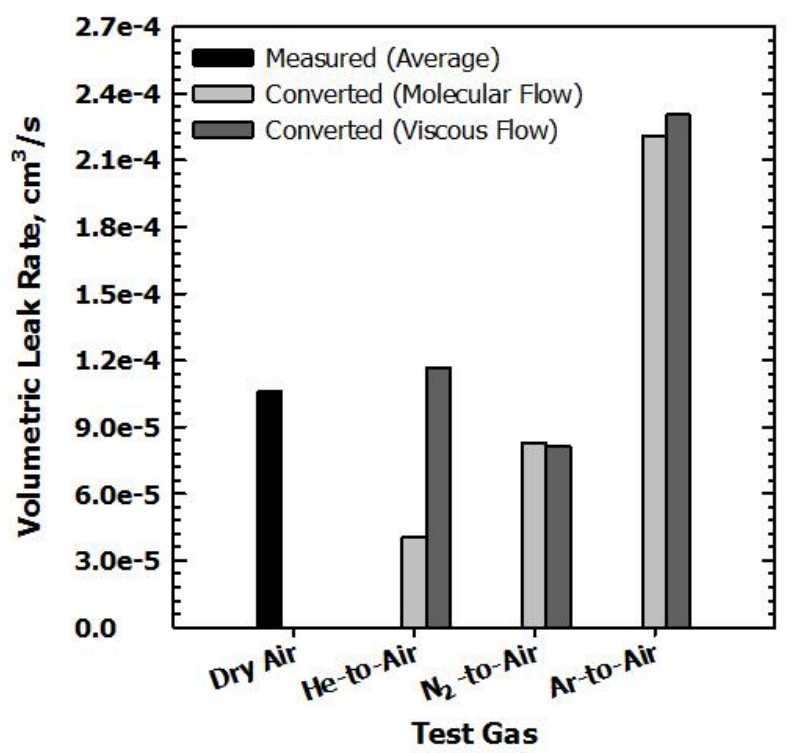

Figure 6. Experimental average volumetric air leak rate at NTP compared to projected air leak rates computed by standard conversion of measured helium, nitrogen, and argon leak rates assuming molecular and viscous flow.

These findings supported the assumption that the standard conversion factors for the viscous and molecular flow regimes were not applicable to a silicone elastomer O-ring whose leak rate was dominated by permeation. Since there is no uniform method of converting the helium measurements to the gas of interest, using a helium leak detector and applying a standard conversion factor cannot be used to accurately determine the air (or other gas) leak rate for this type of seal. Nor can another tracer gas be used in this fashion to verify the air leak rate. If molecular and viscous flow conversion factors are applied to convert a measured tracer gas leak rate, the underlying assumptions must be fully understood to prevent the acceptance of invalid data in spaceflight hardware verification.

\section{Effect of Pressure Boundary Conditions}

In the second test series, the interior volume of the test fixture was pressurized with dry air, while the downstream pressure was continuously adjusted to maintain a constant differential pressure across the test article. The selected differential pressures ranged from 34.5 to $137.9 \mathrm{kPa}$ and up to six different upstream pressures for each differential pressure were tested based on the limitations of the pressure transducers.

The results for each pressure combination are plotted in Fig. 7, along with the average leak rate of the test article with respect to differential pressure. Not surprisingly, the differential pressure significantly influenced the leak rate of the test article. The average leak rate increased linearly with differential pressure and by a factor of 4 from $34.5 \mathrm{kPa}$ to $137.9 \mathrm{kPa}$ (differential pressure).

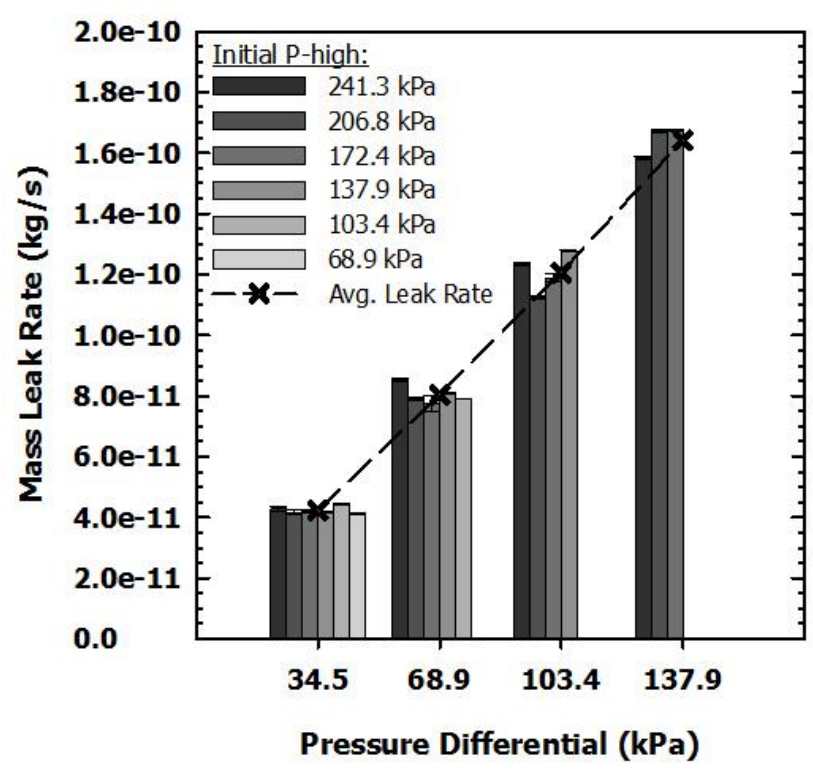

Figure 7. Air leak rates for a silicone elastomer O-ring at room temperature under various pressure boundary conditions. Initial $P$-high represents the initial pressure of the internal volume. Downstream pressure was controlled to maintain a constant differential pressure. Error bars represent measurement uncertainty.

The difference in measured leak rate due to change in the interior volume gas pressure (initial P-high in Fig. 7) was also investigated and determined to be insignificant. While the effect of the absolute pressure did not appear to contribute to changes in leak rate, greater values of differential pressure may have caused increased experimental scatter. The standard deviation of the measured leak rates at a differential pressure of $34.5 \mathrm{kPa}$ was $1.08 \times 10^{-12} \mathrm{~kg} / \mathrm{s}$. For a differential pressure of $103.4 \mathrm{kPa}$, the standard deviation increased by a factor of 6 . Due to the limited number of tests, and decrease in number of tests with increase in 
differential pressure (due to limitations of the transducers) it is difficult to isolate the cause of this scatter. Each test, regardless of pressure conditions, was run with identical controller settings. It is possible that at higher differential pressures, controller settings were not optimal, potentially affecting repeatability of the results.

Overall, the results suggested that the differential pressure was a dominant factor in the leak rate of the test article. Providing the differential pressure is representative of operating conditions, the pressure decay method can be used to accurately measure the air leak rate of similar gas pressure sealing systems using pressure conditions different from those in space.

\section{SUMMARY}

In light of deep space exploration goals for future spaceflight missions, it is critical to verify the performance of spaceflight hardware. When test methods are used for verification purposes, it is necessary to ensure that the methods selected are representative of the operational conditions of the hardware under test and to understand the effect on the test results of those parameters that cannot be directly simulated.

In this study, two test methods for verifying the air leak rate of spacecraft sealing systems were investigated: the use of flow dependent conversion factors with helium leak detector results and the pressure decay method with various boundary conditions. Two series of tests were completed using a modified pressure decay method to determine the leak rate of a silicone elastomer O-ring. All tests were completed at or near room temperature.

In the first test series, the pressure conditions were constant between tests, but four different test gases were used: helium, nitrogen, dry air, and argon. Using standard conversion factors for the viscous and molecular flow regimes, the helium leak rate was converted to respective nitrogen, dry air, and argon leak rates. The experimental results were compared to the calculated results and showed that the helium leak rate of a permeationlevel leak could not be accurately converted based on these two flow regimes. For the system tested in this study, the leak rate values were underpredicted for molecular flow and generally overpredicted for viscous flow. In a similar fashion, the volumetric flow rates for nitrogen and argon were converted to air leak rates and compared to the measured leak rate of air. Neither test gas correctly represented the air leak when converted using viscous or molecular flow. Hence, caution must be used when selecting the helium (tracer gas) leak detector method to verify the air leak rates of spaceflight sealing systems so as not to produce erroneous results.

In the second test series, the pressure boundary conditions of the pressure decay method were examined. Four unique differential pressures, with up to six different initial upstream pressures were tested. The downstream pressure was controlled to maintain a constant differential pressure throughout the duration of the test. Dry air was utilized as the test gas. As expected, the leak rate of the test article was directly related to the differential pressure. Over the limited range tested, the upstream pressure had an insignificant effect on the measured leak rate value provided that the differential pressure remained constant. This suggested that the pressure decay method can be used to measure the air leak rate under various pressure conditions if the differential pressure matches the expected inspace operational value.

On the whole, the findings of this study highlight the importance of fully understanding the applicability and limitations of the test method selected for verification of spaceflight hardware. Incorrectly applying a test method, or not completely representing the functional operations may lead to inaccurate results and have significant implications for any spaceflight mission.

\section{ACKNOWLEDGMENTS}

Support for this work was provided by the National Aeronautics and Space Administration under contract NNC13BA10B.

\section{REFERENCES}

[1] National Aeronautics and Space Administration, NASA Systems Engineering Handbook, Tech. Rep. NASA/SP2007-6105 Rev1, NASA, Washington, D.C., December 2007.

[2] National Aeronautics and Space Administration, International Space Station Program Qualification and Acceptance Environmental Test Requirements, No. SSP 41172 Rev. U, 28 March 2003.

[3] National Aeronautics and Space Administration, Constellation Program Environmental Qualification and Acceptance Testing Requirements (CEQATR), No. CxP 70036 Rev. C, 05 October 2009.

[4] Underwood, S. and Lvovsky, O., Implementation of Leak Test Methods for the International Space Station (ISS) Elements, Systems and Components, Proceedings of the 6th International Symposium on Environmental Testing for Space Programs, Noordwijk, Netherlands, 12-14 June 2007.

[5] Dunlap, Jr., P. H., Daniels, C. C., Wasowski, J. L., Garafolo, N. G., Penney, N., and Steinetz, B. M., FullScale System and Testing Methodology for Quantifying Leak Rates of Docking System Seals for Space Applications, Proceedings of the 45th AIAA/ASME/SAE/ASEE Joint Propulsion Conference \& Exhibit, No. AIAA 2009-5319, Denver, CO, 2-5 August 2009.

[6] Wasowski, J. L., Penney, N., Garafolo, N. G., and Daniels, C. C., Leak Rates of a Candidate Main Interface Seal at Selected Temperatures, Proceedings of the 45th AIAA/ASME/SAE/ASEE Joint Propulsion Conference \& Exhibit, No. AIAA 2009-5320, Denver, CO, 2-5 August 2009.

[7] Garafolo, N. G. and Daniels, C. C., An Experimental Investigation of Leak Rate Performance of a Subscale Candidate Elastomer Docking Seal, Proceedings of the 46th AIAA/ASME/SAE/ASEE Joint Propulsion Conference \& Exhibit, No. AIAA 2010-6907, Nashville, TN, 25-28 
July 2010.

June 2010.

[8] Penney, N., Wasowski, J.L., and Daniels, C.C., Temperature and Atomic Oxygen Effects on Helium Leak Rates of a Candidate Main Interface Seal, Proceedings of the 46th AIAA/ASME/SAE/ASEE Joint Propulsion Conference \& Exhibit, No. AIAA 2010-6986, Nashville, TN, 25-28 July 2010.

[9] Daniels, C.C., Wasowski, J.L., Panickar, M.B., and Smith, I.M., Leak Rate Performance of Three Silicone Elastomer Compounds after Ground-Simulated and On-Orbit Environmental Exposures, Proceedings of the 3rd AIAA Atmospheric Space Environments Conference, No. AIAA 2011-3823, Honolulu, HI, 27-30 June 2011.

[10] Garafolo, N. G. and Daniels, C. C., Experimental Investigation of Leak Rate Performance of a Subscale Composite Elastomer-Retainer Docking Seal, Journal of Spacecraft and Rockets, Vol. 50, No. 3, 2013, pp. 709-714.

[11] LACO Technologies, Salt Lake City, Utah, LACO Technologies Technical Reference Guide: Leak Testing, http://www.lacotech.com/Specifications.aspx. Accessed: 2 December 2016.

[12] Bialous, A., Peters, P., and Stone, E., Leakage Testing Handbook, Tech. Rep. NASA-CR-106139, S-69-1117, General Electric Research and Development Center, Schenectady, New York, July 1969.

[13] Van Atta, C. M., Vacuum Science and Engineering, McGraw-Hill, New York, 1965, pp. 1-62.

[14] Chambers, A., Fitch, R.K., and Halliday, B.S., Basic Vacuum Technology, Institute of Physics Publishing, Philadelphia, PA, 2nd ed., 1998, pp. 143-151.

[15] Smith, I. M., Daniels, C. C., Dunlap, Jr., P. H., and Steinetz, B. M., Performance of Sub-scale Docking Seals under Simulated Temperature Conditions, Proceedings of the 44th AIAA/ASME/SAE/ASEE Joint Propulsion Conference \& Exhibit, No. AIAA 2008-4713, Hartford, CT, 21-23 July 2008.

[16] Garafolo, N. G. and Sawyer, S. D., A Computational Prediction of the Permeation Leak Rate Performance of a Candidate Space Seal, Proceedings of the 49th AIAA Aerospace Sciences Meeting, No. AIAA 2011-425, Orlando, Florida, 4-7 January 2011.

[17] Garafolo, N. G. and Daniels, C. C., The Mass Point Leak Rate Technique With Uncertainty Analysis, Research in Nondestructive Evaluation, Vol. 25, No. 2, 2014, pp. 125149.

[18] Daniels, C.C., Braun, M.J., Oravec, H.A., Mather, J.L., and Taylor, S.C., Leak Rate Quantification Method for Gas Pressure Seals with Controlled Pressure Differential, Proceedings of the 51st AIAA/SAE/ASEE Joint Propulsion Conference \& Exhibit, No. AIAA 2015-4231, Orlando, Florida, 27-29 July 2015.

[19] Garafolo, N. G. and Daniels, C. C., Comprehensive Mass Point Leak Rate Technique. Part I: Methodology with Uncertainty and Experimental Error Analysis, JSNDI/ASNT Fourth Japan - US Symposium on Emerging NDE Capabilities for a Safer World, Maui, Hawaii, 7-11 